Meta

Journal des traducteurs

Translators' Journal

\title{
La traduction en malgache des conventions internationales
}

\section{Raphaël Rajaspera}

Volume 43, numéro 3, septembre 1998

URI : https://id.erudit.org/iderudit/003910ar

DOI : https://doi.org/10.7202/003910ar

Aller au sommaire du numéro

Éditeur(s)

Les Presses de l'Université de Montréal

ISSN

0026-0452 (imprimé)

1492-1421 (numérique)

Découvrir la revue

Citer cet article

Rajaspera, R. (1998). La traduction en malgache des conventions

internationales. Meta, 43(3), 380-392. https://doi.org/10.7202/003910ar

\section{Résumé de l'article}

La traduction en malgache des conventions internationales comportait autrefois quelques pièges sémantiques et diplomatiques plus ou moins importants. Actuellement encore, elle soulève des problèmes de style, d'interprétation des termes et, dans certains cas, de communication avec les lecteurs. 


\title{
LA TRADUCTION EN MALGACHE DES CONVENTIONS INTERNATIONALES
}

\author{
RAPHAËL RAJASPERA \\ Université d'Antananarivo, Antananarivo, Madagascar
}

\begin{abstract}
Résumé
La traduction en malgache des conventions internationales comportait autrefois quelques pièges sémantiques et diplomatiques plus ou moins importants. Actuellement encore, elle soulève des problèmes de style, d'interprétation des termes et, dans certains cas, de communication avec les lecteurs.
\end{abstract}

\begin{abstract}
The translation of international agreements into the malagasy language formerly contained some semantic and diplomatic snares that were more or less important. Even nowadays, it raises problems concerning style, interpretation of terms and, in some case, communication with the readers.
\end{abstract}

Depuis la deuxième moitié du XIX $\mathrm{X}^{\mathrm{e}}$ siècle, Madagascar a entretenu avec l'étranger des relations diplomatiques attestées par des traités, des conventions et des accords, dont nous tirerons ici quelques citations, établis sur la base de textes écrits dans une des langues européennes et de leur traduction en malgache :

- lettre adressée par le gouverneur de La Réunion à Radama II, lors de l'accession de ce dernier au trône, avec une traduction en malgache (30 décembre 1861);

- traduction en malgache des négociations entamées par le gouvernement de Tananarive avec le gouvernement français (documents diplomatiques, affaires de Madagascar, du 26 mars 1882 au 8 janvier 1883);

- lettres commentant certains articles du traité franco-malgache du 17 décembre 1885 : original en français écrit à bord de la Nä̈ade et copie de la lettre d'interprétation avec traduction en malgache de cette lettre (9 janvier 1886);

- copie de la lettre d'interprétation de la lettre Patrimonio-miot, avec traduction en malgache de cette lettre (17 décembre 1885);

- traité de paix, d'amitié et de commerce entre l'Angleterre et Madagascar, texte en anglais et en malgache (27 juin 1865);

- traité de commerce, d'amitié et de paix entre les États-Unis d'Amérique et Madagascar (texte signé par les plénipotentiaires, original en anglais américain et en malgache, 14 février 1867);

- texte ratifié par le président des États-Unis (original en anglais et en malgache, 24 janvier 1868);

— protocole de la conférence tenue à Washington entre les plénipotentiaires malgaches et américains (original en malgache et en anglais, 12 mars 1883);

- convention d'amitié, de commerce et de navigation conclue entre l'Italie et Madagascar (texte signé par les plénipotentiaires, original en malgache et en italien, 6 juillet 1883);

Meta, XLIII, 3, 1998 
- traité de paix et de commerce entre l'Allemagne et Madagascar (texte en allemand, en anglais et en malgache, 15 mai 1883);

— projet de traité de paix et d'amitié entre la Suède, la Norvège et Madagascar (19 janvier 1885).

Mais parmi ces conventions diplomatiques, aucune ne semble avoir eu dans la passé des conséquences aussi durables que le traité de paix du 17 décembre 1885 entre la France et Madagascar ${ }^{1}$, lequel a soulevé, en malgache, des problèmes de traduction d'une portée historique considérable.

De nos jours, nombreux sont les traités, accords, conventions et protocoles d'accords internationaux publiés en français et en malgache dans le Journal Officiel de la République Malgache et le Journal Officiel de la République démocratique de Madagascar. Parmi eux, nous citerons les accords de coopération économique et technique entre la République malgache et le gouvernement de la République socialiste de Roumanie (signés le 25 mai 1970, ratifiés et promulgués le 18 août 1973) et les accords de coopération économique et technique entre le gouvernement de la République malgache et le gouvernement de l'URSS (faits le 31 décembre 1974 et promulgués le $1^{\text {er }}$ novembre 1975).

Et nous nous poserons les questions suivantes : 1) Quel est le rôle des traductions malgaches des conventions diplomatiques entre Madagascar et d'autres nations ? 2) Quelles étaient les difficultés particulières des traductions anciennes ? 3) Qui a traduit et comment les traductions malgaches anciennes et actuelles sont-elles reçues par les lecteurs ou le public?

\section{LE RÔLE DES TRADUCTIONS MALGACHES DES CONVENTIONS DIPLOMATIQUES ENTRE MADAGASCAR ET D'AUTRES NATIONS}

\section{Historique}

$\mathrm{Au} \mathrm{XIX}^{\mathrm{e}}$ siècle, les conventions diplomatiques passées entre Madagascar et d'autres nations devaient être accompagnées de leur traduction en malgache. Il en était toujours ainsi, pour permettre aux autorités malgaches, qui étaient unilingues ou supposées telles, de participer en connaissance de cause aux négociations entre les plénipotentiaires malgaches qu'elles avaient accrédités et les plénipotentiaires étrangers.

Généralement, les textes diplomatiques étaient traduits de l'anglais (ou du français) au malgache, et même la convention d'amitié, de commerce et de navigation entre l'Italie et Madagascar (6 juillet 1883) ou le traité de paix et d'amitié entre la Suède, la Norvège et Madagascar (19 janvier 1885) devaient passer par l'intermédiaire d'une traduction anglaise pour être finalement retraduits en malgache. Le besoin de traductions malgaches était tel que la version malgache du traité de paix du 17 décembre 1885, rédigé en français, devait fatalement servir de version officielle pour les autorités malgaches, le premier ministre malgache, Rainilaiarivony, ignorait le français.

Entre 1960 et 1973, il n'y a pas eu de traduction malgache des conventions entre Madagascar et d'autres nations. Seul le texte français paraissait au Journal Officiel. En effet, le français a été, et reste encore, la langue de travail de la République malgache dans ses rapports avec l'étranger.

C'est à partir de 1973 qu'une traduction malgache a été jugée nécessaire en matière de la ratification des traités, pour faire foi sur le plan national. Ainsi, elle est destinée à informer le public malgache sur les conventions, traités et accords unilatéraux, bilatéraux et multilatéraux entre Madagascar et le monde extérieur. Ces derniers sont promulgués et «vulgarisés» au Journal Officiel bilingue, en français et en malgache : ceci, bien entendu, parce que les résultats des négociations concernent le peuple malgache et qu'ils ont, dans 
leur application, un impact considérable sur l'idéologie et la vie nationales (vie politique, économique, sociale, culturelle, etc.).

\section{LES DIFFICULTÉS LINGUISTIQUES PARTICULIÈRES DES TRADUCTIONS MALGACHES DES CONVENTIONS DIPLOMATIQUES ANCIENNES}

\section{Quelques exemples}

$\mathrm{Au} \mathrm{XIX}{ }^{\mathrm{e}}$ siècle, il n'était pas toujours aisé, pour établir les textes bilingues des conventions diplomatiques, de faire la reconnaissance parfaite de l'unité lexicale à traduire, puis de faire coïncider le contenu des mots étrangers et des expressions malgaches, ou de faire la traduction littérale de la totalité de l'information conceptuelle contenue dans l'original : les concepts utilisés n'étaient pas toujours formulables d'une façon nette et précise dans la langue malgache d'autrefois. Souvent, les traducteurs essayaient de compenser les insuffisances du vocabulaire malgache par une modification de la terminologie étrangère ou par des déplacements sémantiques, parfois par des circonlocutions.

En effet, il existait un certain nombre de termes dans les traités et les conventions entre les nations étrangères et Madagascar, qui, dès la langue de départ, avaient un sens technique très précis, mais qui, à l'époque, étaient très difficiles à rendre en malgache, faute d'expressions consacrées équivalentes. Ce sont, par exemple, les concepts de paix, bail emphytéotique, protectorat, représentation, relations extérieures.

Le mot paix était traduit de façon variable. Certaines conventions diplomatiques avec l'Allemagne, les États-Unis et la France portaient les titres : Friedensvertag, Treaty of Peace, Traité de paix, rendu en malgache par fanekena tsy hiady (litt. «accord pour qu'il n'y ait pas état de guerre»). Mais le terme paix se confondait avec fihavanana (amitié), qui traduisait, en même temps que fisakaizana, le mot friendship, à l'intérieur d'un énoncé tel que : ireo Fanjakana roa tonta lehibe mifaneky ireo dia milaza marina fa hisy fihavanana mafy tsy azo ravana, sy fisakaizana marina tokoa amin'Izy roa tonta sy ny mpandova Azy, sy Izay handimby Azy mandrakizay, ary tsy hiady (Fanekena tsy hiady, sy fihavanana ary fivarotana amy ny America sy Madagaskara, 14 février 1867)2 ${ }^{2}$ En effet, on n'avait pas encore trouvé un terme consacré comme fanekem-pihavanana (traité de paix) (Malzac 1893 : Dictionnaire français-malgache), ou comme fandriampahalemana (paix) actuellement utilisé dans un énoncé tel que : «l'Océan Indien doit être zone de paix» Ny Oseana Indiana dia tokony ho faritry ny fandriampahalemana.

L'expression bail emphytéotique, dans le traité de paix entre la France et Madagascar du 17 décembre 1885, était traduite d'une façon qui, selon Faranirina Rajaonah, pouvait «prêter à confusion» ou «permettre une interprétation très large»: fanofanana maharitra (litt. «location de longue durée $\left.{ }^{3} »\right)$. Le même terme devait remplacer le mot vente, pour annuler, à l'endroit des ressortissants étrangers résidant à Madagascar, le droit de propriété stipulé par l'article IV du traité anglo-malgache de 1865. Or, une lettre appendice du ministre plénipotentiaire Patrimonio et du contre-amiral Miot précisait : «à l'article 6, l'expression bail emphytéotique signifie un bail spécial d'une durée de 99 ans et renouvelable au gré des parties» (9 janvier 1886). La traduction posait donc des problèmes gênants d'interprétation au moment de l'application du traité.

Cependant, «tant que les différences de traduction ne concernent que des clauses de portée restreinte, les problèmes sont limités [...] À partir du moment où elles mettent en cause les points essentiels, de graves complications surgissent ${ }^{4} \gg$. De sérieux problèmes concernent particulièrement les cas où le texte en langue étrangère et sa traduction malgache n'ont pas la même signification fondamentale : ainsi, la traduction malgache du texte français du traité de paix du 17 décembre 1885 avait été tournée de telle façon que les 
autorités malgaches n'avaient pas compris le message essentiel dans le sens conforme à l'original français.

En effet, l'enjeu de ce traité était le protectorat français à établir sur Madagascar; l'idée était implicite dans les clauses de l'acte sans que le terme protectorat ait été mentionné une seule fois. La non-production du mot protectorat dans le texte français et, bien entendu, dans la traduction malgache du traité relève, semble-t-il, d'un procédé de masquage : le mot était volontairement omis en raison de ses connotations vexantes pour les Malgaches, qui ne voulaient absolument pas d'une mainmise de la France sur Madagascar. Et les expressions françaises qui définissaient le protectorat tout au long du traité avaient été traduites en malgache de manière inadéquate au point que les autorités malgaches s'étaient trompées sur la signification exacte des articles essentiels.

Par exemple, dans l'article 1 du traité, il est stipulé que «le Gouvernement de la République représentera Madagascar dans toutes ses relations extérieures ${ }^{5}{ }$ ou, dans l'article 2, qu'«... un Résident représentera le Gouvernement de la République aux relations extérieures de Madagascar ${ }^{6} »$. Or, dans les traductions malgaches correspondantes, le terme hitandrina (surveillera, prendra soin $\mathrm{de}^{7}$ ) ne saurait pas signifier valablement représentation. L'idée de représentation, qui n'est pas contenue dans hitandrina, aurait pu être rendue par hisolo toerana (remplacera), ou, tout au moins, par la même forme hitandrina, mais avec le sens de diriger, présider, comme il a été fait dans l'article 12 du même traité : ny mpanjaka ny Madagascar hitandrina ny fitondram-panjakany amy ny ati-taniny manerana ny nosy manontolo, toraky ny tamy ny lasa ${ }^{8}$. C'est l'idée de représentation, telle qu'elle est signifiée par le texte français où elle indique la substitution du haut représentant français au gouvernement malgache dans l'exercice de certaines activités gouvernementales, qui est atténuée par un euphémisme dans la traduction. C'est donc la traduction tout entière des articles essentiels du traité qui est mise en cause ici.

Et l'on comprend alors comment la divergence fondamentale de signification entre le texte français et le texte malgache avait eu pour conséquence de fausser dès le départ l'interprétation malgache de la traduction de «[la France] représentera Madagascar dans toutes ses relations extérieures» ou de "présidera aux relations extérieures de Madagascar».

Dans le texte préparé en français, relations extérieures avait une signification bien précise pour le législateur, les juristes et les utilisateurs français. Cette expression était utilisée de la même façon dans tous les traités de protectorat passés par la France. Mais la traduction malgache de cette unité d'énoncé, en reformulant le signifié de l'énoncé premier, soulevait une ambiguïté .

En effet, dans une perspective générative et transformationnelle, la traduction malgache de relations extérieures (firaharahana amy ny Fanjakana any ivelany «négociations avec les gouvernements, à l'extérieur» ou «négociations avec les gouvernements étrangers») est un fragment d'énoncé appartenant à une structure de surface équivoque qui serait dominée par deux structures profondes sous-jacentes de sens tout à fait différents :

1) la France remplacerait Madagascar dans ses «relations avec l'extérieur», avec les gouvernements étrangers ( $i$ France dia hisolo toerana an'i Madagascar amin'ny fifandraisany amin'ny Fanjakana an'ivelany), en ce sens que la France contrôlerait toutes les affaires étrangères et les relations diplomatiques, selon le régime du protectorat;

2) la France représenterait Madagascar dans ses «relations à l'extérieur» (i France dia hisolo toerana an'i Madagascar amin'ny fifandraisany an'ivelany), dans le sens d'une représentation diplomatique «à l'étranger». 
Sémantiquement parlant, rien ne permettait a priori aux Malgaches de choisir plutôt une interprétation qu'une autre, étant donné la façon dont la traduction avait été formulée. Même le contexte, où le mot protectorat n'était pas produit, n'aurait pas pu les tirer de la perplexité, l'ambiguïté de la performance renvoyant ici au caractère double du modèle de compétence.

Ainsi, la traduction des anciens traités et conventions d'une langue européenne en malgache posait, à l'époque, des problèmes d'interprétation particulièrement délicats, lorsque, dans les textes à traduire, la moindre ligne de chaque passage était déjà susceptible de contenir un piège sémantique et diplomatique et que la traduction malgache en déformait le sens.

\section{LES TRADUCTEURS EN MALGACHE DES CONVENTIONS INTERNATIONALES FACE À LEURS LECTEURS}

\section{La responsabilité des traducteurs}

La qualité de la traduction est sans doute une des conditions nécessaires de la communication du message au public destinataire. Dans la mesure où son étude amène à prendre en considération la gamme des moyens linguistiques par lesquels l'énoncé est plus ou moins assumé, il semble désormais important, à propos des traductions malgaches des conventions diplomatiques anciennes et récentes, de se demander quels sont les rapports entre traducteurs et public destinataire de la traduction : qui a traduit et comment le message traduit est-il reçu ?

Tant que les traités de paix, d'amitié, de commerce et les conventions économiques et techniques sont conçus dans le même esprit dans les textes en langue étrangère et en malgache, théoriquement, on peut penser que les problèmes les plus épineux sont aplanis et qu'il n'y a plus rien à demander aux responsables de la traduction. Du reste, ceux-ci s'effacent et restent dans l'anonymat devant les actes officiels qu'ils traduisent et que signent les parties contractantes représentées par les plénipotentiaires des pays intéressés.

Mais des problèmes surgissent lorsqu'on met en regard des textes parallèles dans les deux langues et que des différences de signification et de valeur s'imposent avec évidence au niveau des expressions ou du contenu global. Une foule de questions se posent alors à propos des traductions malgaches de ces conventions et traités, qui étaient susceptibles de soulever de sérieux problèmes d'interprétation ou qui restent encore discutables.

\section{PAR EXEMPLE, QUI A TRADUIT LE TRAITÉ DU 17 DÉCEMBRE 1885 ET COMMENT LA TRADUCTION A-T-ELLE ÉTÉ REÇUE?}

En confrontant le texte français et le texte malgache du traité du 17 décembre 1885, on peut se demander quel était l'effet de l'ambiguïté relative à la traduction malgache par rapport à l'original français.

Déjà, dès le texte français, l'omission volontaire du mot protectorat afin de ne pas braquer le premier ministre Rainilaiarivony, qui n'en voulait pas et qui aurait continué une lutte que les Français ne désiraient pas poursuivre, avait une influence sur le choix des termes de la traduction malgache et avait une grande portée : les Français avaient ainsi pensé, d'une façon très diplomatique, pouvoir parvenir à mettre en place un système de contrôle du gouvernement malgache sans que celui-ci, manœuvré par les termes du traité, s'y refusât. Une fois dans la place, il leur était possible de poursuivre une politique d'empiètements systématiques sur les activités du gouvernement malgache, comme cela s'était passé dans les autres protectorats. 
Il suffisait, dans le texte malgache de l'article 1, de quelques mots comme mitandrina (surveiller, prendre soin de) pour introduire dans la traduction une distorsion de la signification et atténuer le sens fondamental du texte français. Et la question est de savoir : «Qui avait intérêt à traduire ainsi ?» ou «Qui avait traduit ?».

Était-ce Digby Willoughby, sujet britannique, généralissime des troupes merina, accepté par l'amiral français Miot pour négociateur et représentant de la Reine dans la signature du traité ? Sa réputation de grand aventurier qui ne s'embarrassait pas de scrupules, que les historiens lui attribuent, aurait incliné à faire penser que sa traduction malgache du texte français avait été aménagée pour rendre le traité acceptable par le premier ministre.

Était-ce Rainandriamampandry-Rabezandrina, gouverneur de Tamatave, qui, étant instruit, connaissait l'anglais et le français en plus de sa langue maternelle ? Sa réputation de patriote intelligent aurait amené à croire que sa traduction comportait une simple impropriété dont il se serait aperçu après coup qu'elle possédait une signification de laquelle il était possible de tirer profit, d'un point de vue malgache. Ou bien, d'emblée, l'équivoque de la traduction avait-elle été voulue ainsi, du côté malgache représenté par les traducteurs présumés Rainizanamanga, Rainandriamampandry et Willoughby, pour permettre de limiter au maximum la portée d'application du traité ?

Comment le texte malgache a-t-il été compris par les autorités malgaches ?

La traduction semblait déjà façonner un certain modèle d'interprétation à l'usage de ceux qui étaient les destinataires du texte malgache. Ainsi, si, pour les Européens habitués à jongler avec les termes de colonie, protectorat, la phrase «les Malgaches à l'étranger sont placés sous la protection de la France» a un sens politique très précis ${ }^{10}$, par contre, pour les Malgaches de l'époque, le terme choisi pour traduire protection (fiarovana) ne pouvait évoquer que l'idée de «protection contre un danger» et non l'idée de protectorat.

Et, dans la traduction, la signification de certains mots, qui apparaît comme une impropriété voulue, soulevait des interprétations amenant à des situations désormais compliquées. Par exemple, là où la France entendait prendre la place du gouvernement malgache pour mener toute la politique étrangère de Madagascar, les Malgaches, dont le premier ministre, avaient compris la version «ny Gouvernement ny Repoblika hitandrina ny firaharahana ataony Madagascar amy ny Fanjakana any ivelany» comme signifiant que la France se chargerait de leurs «relations à l'extérieur» (représentations diplomatiques à l'étranger), mais que les négociations menées avec les puissances étrangères à l'intérieur du territoire relevaient de leur compétence : la délivrance de l'exequatur aux consuls étrangers devait alors entrer dans le domaine de l'administration intérieure du pays, car les consuls avaient à exercer leurs fonctions sur le territoire malgache même, et non «à l'étranger» ${ }^{11}$. Il y avait malentendu entre les parties contractantes. Or, il était bien précisé, dès le départ, que le texte français et le texte malgache faisaient également foi.

Une mise en parallèle des deux écrits, en français et en malgache, appelle donc les observations suivantes : les problèmes de la traduction malgache des traités à cette époque où manquait déjà la connaissance de ce que pouvait être, de façon générale, la langue technique des conventions diplomatiques étaient tributaires :

1) de l'importance des impropriétés et des contresens commis dans la traduction;

2) des intentions politiques secrètes des traducteurs; et

3) des divergences d'interprétation dans la façon dont le message pouvait être perçu par les parties contractantes. 


\section{LA VULGARISATION DES TRADUCTIONS MALGACHES DES CONVENTIONS INTERNATIONALES ACTUELLES}

On pourrait s'étonner de constater encore que sont relativement peu nombreux les lecteurs qui lisent directement dans le Journal Officiel les traductions malgaches des conventions internationales actuelles et que rares aussi sont ceux qui lisent à la fois l'original et la traduction. Ces constatations soulèvent des questions sur : 1) la qualité des traductions; 2) la formation antérieure des traducteurs; 3 ) les difficultés de la langue de la traduction et la façon dont le public peut recevoir le message en malgache.

\section{LA QUALITÉ DES TRADUCTIONS ACTUELLES}

Les meilleures traductions malgaches parues au J.O. sont celles qui rendent le sens des textes législatifs et réglementaires de la langue de départ (le français) d'une façon compréhensible, précise et dans un style conforme au génie de la langue malgache. Certains traducteurs semblent y être parvenus.

Mais la traduction des conventions, des traités et des accords internationaux a soulevé, et soulève encore, des problèmes particulièrement ardus au point de vue du choix du vocabulaire malgache nécessaire pour rendre les termes techniques et au point de vue du style.

Les quelques traductions connues des conventions actuelles ont été très littérales, presque mot pour mot. Certaines, à caractère assez technique, semblent relativement intelligibles dans la mesure où elles peuvent encore rendre compte des réalités auxquelles elles réfèrent. Si nous relevons tel énoncé, extrait du J.O.R.D.M. du 2 janvier 1980 :

S'agissant des paiements des marchandises dont les prix seront à fixer sur la base des cours mondiaux, ils s'effectueront en monnaies librement convertibles conformément aux législations nationales en cours

et si nous comparons à sa version malgache :

Ny amin'ny fanefana ny vidin'ny entam-barotra, izay ho ferana arakaraka ny sandam-bola eran-tany, dia hatao amin'ny vola azo atakalo malalaka araka ireo lalàna manan-kery ao amin'ny firenena izy ireny ${ }^{12}$

nous pouvons encore y voir une forme de traduction juxtalinéaire susceptible de paraître tout à fait convenable pour des lecteurs bilingues ayant une instruction assez poussée pour la comprendre et une expérience des opérations monétaires.

D'autres traductions interlinéaires subissent spontanément, semble-t-il, l'influence de la langue des textes en français dont elles essaient de rendre le plein sens : nous y retrouvons des structures parallèles de phrases en français et en malgache, régulièrement comparables; l'enveloppe extérieure de chaque énoncé est presque identique ou aisément superposable pour un même message pensé dans les deux langues ${ }^{13}$. Ces traductions, qu'on pourrait qualifier soit de parallèles, soit de directes, selon le cas, voudraient conserver à tout prix les mêmes tours d'une langue à l'autre, dans le souci, légitime en soi, de rester fidèles au texte original français.

Mais la question est de savoir si elles ont toutes la possibilité de rendre compte des réalités exprimées ou si elles les faussent au contraire, et si elles peuvent être comprises par tout le public des lecteurs exactement comme les traducteurs comprennent ce qu'ils veulent eux-mêmes dire. Car rien ne paraît dérouter autant un lecteur malgache qu'un ordre des mots peu habituel calqué sur l'ordre des mots français, lorsque ce même lecteur aurait tourné différemment la phrase et lorsqu'il n'arrive pas à suivre la structure grammaticale qu'on lui présente (souvent sous la forme de phrases longues) - ce sont là, du moins, les quelques avis que nous avons recueillis çà et là. Et le malaise est particulière- 
ment sensible dans un pays anciennement bilingue comme Madagascar où la qualité de la traduction se mesure instinctivement non pas à la différence formelle, mais à la ressemblance sémantique de deux textes parallèles en français et en malgache.

\section{LA FORMATION ANTÉRIEURE DES TRADUCTEURS}

Le Journal Officiel bilingue contient les textes législatifs et réglementaires soumis à l'attention du public par chaque ministère. Ceux qui ont rédigé ces textes - ce sont des administrateurs civils, des juristes, des économistes, des financiers, des douaniers, des enseignants littéraires, etc. - les présentent obligatoirement en français et en malgache. Techniciens de l'administration et non traducteurs professionnels, il sont donc de formation assez différente pour qu'il y ait une diversité des styles et, parfois, une certaine fluctuation dans la dénomination malgache des concepts ou dans la terminologie utilisée. Et le problème principal concerne tout particulièrement leur compétence de ce que l'on pourrait appeler la langue malgache des conventions diplomatiques.

En effet, un des problèmes aigus qui se pose aux traducteurs formés dans la culture occidentale, c'est-à-dire longtemps hors du milieu culturel du peuple d'où ils sont issus, c'est qu'ils se sentent mal à l'aise pour formuler dans leur langue maternelle les notions et la pensée juridico-économique qu'ils ont l'habitude de manier en français. Certaines de leurs traductions littérales s'en ressentent, parce que, instruits dans la grammaire française, ils ont tendance à vouloir réorganiser la syntaxe de la phrase malgache selon la ligne des grammaires des langues européennes qu'ils ont étudiées. Bien entendu, s'ajoutent, pour eux, les difficultés à rendre compte de toutes les nuances du malgache dans les domaines très techniques de leurs spécialités.

Mais suffit-il aussi d'être un bon malgachisant pour être à même de traduire en malgache des textes à caractère technique ou juridico-économique ? Or, on peut traduire «les signifiés des terminologies dans la mesure où l'on connaît $[\ldots]$ les techniques auxquelles elles répondent et non pas (seulement) dans la mesure où l'on connaît la langue ${ }^{14}$ ». Car, il est certain que, même si elle est intimement liée à la tradition littéraire du pays, la traduction technique en malgache n'est pas a priori un exercice littéraire : il faut d'abord que les traducteurs sachent appréhender la réalité économique exprimée par le texte français avant de songer à rendre l'idée en bon malgache.

\section{LES DIFFICULTÉS LINGUISTIQUES DE LA TRADUCTION DES CONVENTIONS INTERNATIONALES ACTUELLES ET LE PUBLIC MALGACHE}

Actuellement, les difficultés de la langue malgache à traduire les termes et tournures propres à certaines conventions diplomatiques ne sont pas entièrement surmontées. En effet, la terminologie (surtout dans le domaine économique, commercial et technique) est devenue de plus en plus complexe au fur et à mesure qu'elle change et évolue avec le temps. Constamment, par une consultation attentive des textes économiques et techniques rédigés en français, il devient nécessaire pour les traducteurs de s'assurer que les termes français qu'ils connaissent n'ont pas évolué et ne se sont pas chargés de nouvelles significations. Et certaines formules, à moins d'une initiation très poussée à la question, ne peuvent pas toujours être comprises parce qu'elles relèvent d'un niveau de langue peu accessible : traducteurs et lecteurs butent alors sur les signifiants.

Le langage des conventions internationales actuelles semble avoir déjà un caractère déroutant, dans la mesure où sa terminologie est abstraite, très technique et souvent complexe. En l'absence d'une terminologie unifiée, la traduction malgache elle-même comporte, il faut l'avouer, beaucoup d'incertitudes dans le choix de ses termes. 
On n'est jamais sûr, en effet, d'avoir rendu totalement ni d'avoir fait comprendre les concepts de la langue de départ. Le mot coopération se traduit tantôt par fifarimbonana («action d'agir ensemble», Accord de Coopération Économique et Technique entre Madagascar et la Roumanie, J.O.R.M. du 18/08/1973), tantôt par fiaraha-miasa («action de travailler ensemble», Accord de Coopération Économique et Technique entre Madagascar et l'URSS, J.O.R.D.M. du 01/11/1975), sans que l'on sache quel est le terme unique qui doit être consacré par l'usage.

L'emploi de termes généraux pour traduire des notions pourtant très précises ne facilite pas toujours la compréhension des lecteurs. Est-il certain, en effet, que le public devine dans le mot fitadiavana (litt. «action de chercher»), à l'intérieur du syntagme ny asa kasaina hanaovana fitadiavana (litt. «les travaux conçus pour faire des recherches»), le sens technique très restreint de recherches des gisements miniers ou pétrolifères ou de prospections, dans le contexte des accords de coopération économique et technique entre Madagascar et l'URSS $^{15}$ ? De même, le lecteur peut-il comprendre cette traduction faite à l'aide de termes tout à fait généraux, fampandrosoana ny fahaiza-manao zavatra («façon, action de développer le savoir-faire»), dans une acception plus étroite et plus moderne : «développement dans le domaine des techniques de la matière» (industrielles, agricoles, par exemple; cf. même accord) ?

Quelquefois, les expressions choisies apparaissent comme des euphémismes et ne semblent pas traduire directement les faits. On veut rendre octroi de crédits ${ }^{15}$ par fanomezam-bola («action, fait de donner de l'argent», Accord avec l'URSS). Mais s'agit-il réellement de donner de l'argent, dans le sens d'une aide non remboursable, à fonds perdu ? Ne s'agit-il pas de prêts (fampisamboram-bola) à long terme ou de possibilité d'obtenir des capitaux par suite de la solvabilité que l'on présente, ce qui fait supposer qu'il y ait dettes, créances (trosa, fitrosoana) et crédibilité (fahatokiana), à condition que l'aide dont il s'agit soit remboursable et que la capacité d'endettement ne soit pas saturée ? C'est là que les traducteurs auraient besoin de revoir et leur traduction et la manière dont leur traduction pourra être reçue par le public.

Et l'on pourrait multiplier les exemples, dans ce domaine, des problèmes de traduction, si l'on veut bien que la traduction malgache serve pour le public de vulgarisation des conventions internationales.

L'absence de traduction au J.O., ici, est un élément d'information intéressant dans la mesure où elle montre combien immense est la tâche des traducteurs qui auront à forger un type de discours en malgache, propre aux conventions économiques et commerciales et susceptible d'être perçu par le public auquel il sera destiné : ainsi, certains accords, comme par exemple, la deuxième convention ACP-CEE de Lomé (J.O.R.D.M. du 19/07/1980) ou la convention sur la navigation maritime, passée avec l'URSS (1975) ne sont connus que dans la seule langue de travail, le français, et n'ont pas été traduits en malgache dans le Journal Officiel bilingue.

Et l'on pourrait se demander encore comment traduire en malgache les textes des accords du GATT (1963) où abondent des expressions consacrées et très techniques telles que : relèvement tarifaire, obstacles non tarifaires aux échanges, droits compensateurs, droits anti-dumping, zone de libre-échange, traitement ou clause de la nation la plus favorisée, marges de préférences, etc.; ou encore, les expressions consacrées des négotiations commerciales multilatérales du Tokyo-Round entre les membres du GATT (février 1973) : réduction des droits de douane, traitement différencié, approche sectorielle, American selling price, préférences généralisées, prélèvements fixes et mobiles, contingentements à droits nuls, contingentements à droits partiels, contingentements à plafond, etc. 
Certes, il y a eu des tentatives de traduction d'expressions consacrées (p. ex. : détérioration des termes de l'échange, traitement de la nation la plus favorisée, etc.). Mais ont-elles été satisfaisantes?

On a traduit détérioration des termes de l'échange par fitotonganan'ny vidin'ny akoram-bokatra («chute du prix des produits des récoltes»). Cependant, ce néologisme, intelligible en soi dans son sens littéral pour un Malgache moyen, rend-il aussi bien l'idée de cet écart désastreusement croissant entre pouvoirs d'achat des pays riches et des pays pauvres, entre les prix des matières premières produites et vendues par les pays en voie de développement et les prix des produits finis importés par des pays industrialisés ? Il ne le semble pas.

Certaines formules, utilisées dans le premier accord commercial multilatéral du GATT, telles que clause de la nation la plus favorisée, font difficulté. Il est vrai, elles avaient été à peu près semblablement utilisées dans les traités avec la France, l'Angleterre et l'Allemagne au XIX ${ }^{\mathrm{e}}$ siècle. Cependant, l'expression avait-elle été traduite et comprise dans le sens voulu?

Ainsi, on trouvait: «on the footing of the most favoured nation» (traité de paix, d'amitié et de commerce entre l'Angleterre et Madagascar, 27/06/1865), «comme les sujets de la nation la plus favorisée» (traité franco-malgache, 8/08/1868), «all the rights there enjoyed be the subjects and citizens of the most favored nation» (version anglaise du traité de paix et de commerce entre l'Allemagne et Madagascar, 15/05/1883).

Et les équivalents malgaches étaient donnés à cette époque-là sous la forme : «toraky ny vahoaka avy aminy izay firenena nahazo soa be indrindra ${ }^{16}$ », «toraka izay azon'ny vahoaka sy olona avy amy ny firenena mahazo soa be indrindra ${ }^{17}{ }$, ou encore : «izay avela ataon'ny firenena izay tiana indrindra ${ }^{18}$ ». C'étaient des traduction juxtalinéaires apparemment sans aucun caractère technique. Et l'on se demande si ces traductions avaient été comprises comme il le fallait, au moment de la ratification des traités, puisqu'elles contredisaient de façon flagrante l'article 85 du Code des 305 articles, qui annulait le droit de propriété accordé aux résidents étrangers ${ }^{19}$.

Des problèmes nouveaux se posent pour l'expression moderne traitement de la nation la plus favorisée, parce qu'elle a changé de signification aujourd'hui : elle se limite au domaine commercial et aux tarifs douaniers. Et jusqu'à preuve du contraire, et à moins de trouver plus tard des néologismes appropriés ou des périphrases, on concevrait difficilement une traduction qui exprime avec netteté pour le peuple l'idée contemporaine que tout traitement préférentiel (en avantages commerciaux, fiscaux ou douaniers) qu'une partie contractante accorde à une autre doit être étendue à toutes les autres parties contractantes.

\section{CONCLUSION}

D'une façon générale, les problèmes de traduction des textes de conventions se situent alors à trois niveaux :

1) au niveau des traducteurs-émetteurs : ceux-ci ne peuvent pas traduire convenablement s'ils ne comprennent déjà pas le sens des textes de la langue originale. Pour cela, il faut qu'ils aient assisté aux débats entre parties contractantes et aux séances plénières de travail lors de la signature des conventions ou, tout au moins, qu'ils connaissent bien le sujet, qu'ils s'en soient préalablement imprégnés à l'aide de la documentation nécessaire, à l'aide d'un contexte étendu. En outre, il faudrait qu'ils aient une parfaite maîtrise de leur langue maternelle; 
2) au niveau de la langue de la traduction : les difficultés s'accroissent à mesure que les néologismes techniques de la langue diplomatique, juridico-économique et commerciale, augmentent en quantité et en précision. Faut-il alors multiplier les néologismes malgaches en traduction, au risque de ne pas se les faire accepter ou de ne pas se faire comprendre? Ou faut-il simplifier la traduction en forgeant des périphrases susceptibles d'être comprises et destinées, malgré leur imprécision et leur inexactitude, à entrer dans l'usage actuel, au risque de fausser les réalités exprimées par le texte de la langue de départ?

3) au niveau du public-récepteur: comment lui rendre communicable sa traduction ? Car il faut aussi que le lecteur soit à même de saisir les nuances des textes qu'on lui propose si l'on veut que le meilleur test de la valeur de la traduction malgache soit dans l'accueil que le public lui réserve. En cela, et c'est là l'idéal, il serait souhaitable que la population comprenne l'essentiel des phénomènes économiques qu'elle est en train de vivre, grâce à une alphabétisation juridique et économique intensive.

Aux difficultés de la traduction s'ajoutent les difficultés de compréhension, de la part des lecteurs. D'une part, il y a la barrière notionnelle qu'oppose l'expression des concepts propres aux conventions internationales à caractère diplomatique, économique et technique; d'autre part, il y a cette exigence d'intelligibilité dans la perspective d'une communication optimale avec le public. Une traduction littérale (juxtalinéaire ou interlinéaire) qui essaie en même temps de simplifier au maximum le sens des notions risque, contrairement aux effets escomptés, non seulement de ne pas rendre fidèlement ou suffisamment le texte original, mais aussi, à cause du style, de n'être pas appréciée favorablement par un public difficile, voire simplement de ne pas être lue du tout.

L'autre problème auquel le traducteur se heurte est de savoir comment moderniser la langue malgache des conventions diplomatiques, qu'un public motivé et sensibilisé a le plus besoin de comprendre, mais aussi comment permettre à la population tout entière de comprendre sa traduction : cela suppose (mais ceci n'est plus de son ressort) un effort considérable d'initiation économique et juridique du peuple.

\section{Notes}

1. Archives Nationales de la République Malgache (cotes : DD1, DD2, DD4, DD5).

2. Traduction en français : «Les deux hautes représentations gouvernementales qui concluent ici un accord déclarent solonnellement qu'il y aura pour toujours de bonnes relations fermes et inviolables et une amitié sincère entre les deux parties, leurs héritiers et leurs successeurs, et qu'il n'y aura jamais aucune guerre». (Traité de paix, d'amitié et de commerce entre l'Amérique et Madagascar, 14/02/1867). Texte original anglais du traité : «The high contracting parties solemnly declare that there shall continue to be a firm inviolate peace, and a true and sincere friendship existing between them and their respective heirs and successors for ever without war».

3. Faranirina Rajaonah (1968) : «Attitude malgache face au traité de 1885, d'après le journal de Rainilaiarivony», DES d'histoire, Tananarive.

4. F. Rajaonah, op. cit.

5. Texte malgache : «Ny Governement ny Repoblika hitandrina ny firaharahana rehetra ataony Madagascar amy ny fanjakana any ivelany» (litt. «le gouvernement de la République prendra soin de toutes les négociations menées par Madagascar avec les gouvernements à l'étranger»).

6. Texte malgache : «Résident iray misolo ny Governement ny Repoblika hitandrina ny firaharahana Madagascar amy ny fanjakana any ivelany» (litt. «un Résident représentant le gouvernement de la République prendra soin des négociations menées par Madagascar avec les gouvernements à l'étranger»).

7. C'est, du moins, le sens qu'en donne le dictionnaire du pasteur Ramino : mitandrina = miambina (surveiller), mitaiza (prendre soin, élever).

8. Texte français : «Sa Majesté la Reine de Madagascar continuera, comme par le passé, de présider à l'administration intérieure de toute l'île».

9. Cf. P. Randrianarisoa (1970) : «La diplomatie malgache face à la politique des Grandes Puissances (18821895)», Thèse de doctorat de $3^{\mathrm{e}}$ cycle en histoire, Paris. 
10. F. Rajaonah, op. cit. La traduction malgache de la phrase de l'article était formulée ainsi : «Ny Malagasy izay any amy ny tanim-bahiny dia hapetraka amy ny fiarovany France».

11. En cela, d'ailleurs, Rainilaiarivony se référait à l'alinéa 2 de l'article II et à l'article XII du traité, selon lequel «Sa Majesté la Reine de Madagascar continuera, comme par le passé, de présider à l'administration intérieure de toute l'Île» (cf. P. Randrianarisoa, op. cit., pp. 91-92).

12. Litt. «ce qui concerne les paiements des prix des marchandises, lesquels [prix] seront fixés selon les cours mondiaux des changes, s'effectuera en monnaies librement échangeables conformément aux législations en vigueur dans ces États-là» (exposé des motifs de l'ordonnance portant ratification de l'accord commercial entre le gouvernement de la République démocratique de Madagascar et le gouvernement de la République démocratique allemande, J.O.R.D.M. du 02/01/1980).

13. Voir ces deux énoncés dans les deux langues, que nous avons analysés en unités de traduction (selon la méthode de J.-P. Vinay et J. Darbelnet (1958) : Stylistique comparée du français et de l'anglais, Paris, Didier), de façon à faire apparaître la similitude de l'ordre des mots en LD et en LA :

\section{Énoncé 1}

LD : /«Le Gouvernement de la République Malgache et le Gouvernement de la République Socialiste de Roumanie»/

LA : INy Governemantan'ny Repoblika Malagasy sy ny Governemantan'ny Repoblika Sosialista ao Roumanie/

LD : /animés / du désir de / promouvoir / la coopération / économique / et technique / entre les deux pays

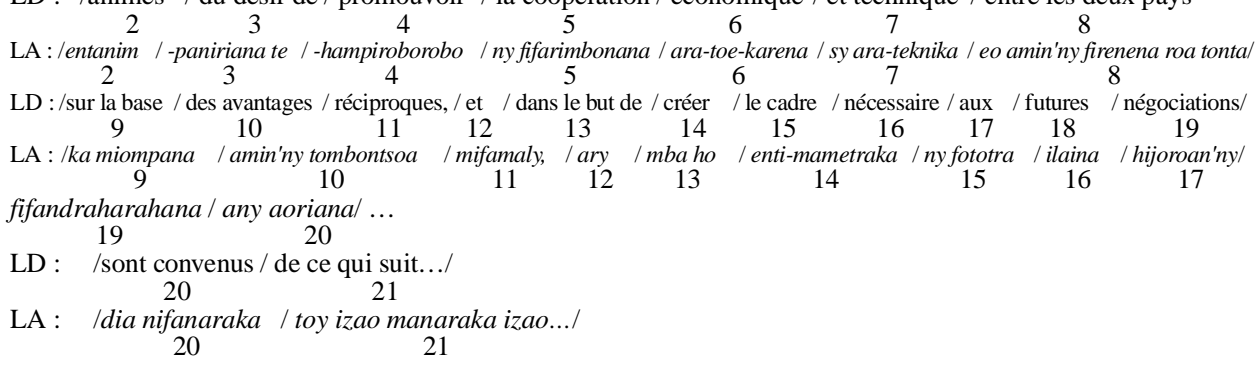

Litt. : «Le Gouvernement de la République Malgache et le Gouvernement de la République Socialiste de Roumanie, soulevés par le désir de faire flamboyer l'action d'agir ensemble au point de vue de l'économie (état de la richesse) et au point de vue technique, entre les deux nations, et attachés aux avantages réciproques, et afin de servir à poser les bases dont on a besoin, sur lesquelles se tiendront les négociations futures [...] sont convenus comme suit...» (Accord de Coopération Économique et Technique entre le Gouvernement de la République Malgache et le Gouvernement de la République Socialiste de Roumanie, J.O.R.D.M. du 18/08/1973).

\section{Énoncé 2}

LD : /Le Gouvernement de la République Malgache et le Gouvernement de l'Union des Républiques Socialistes Soviétiques/ 1

LA : INy Governemantan'ny Repoblika Malagasy sy ny Governemantan'ny Firaisan'ny Repoblika Sosialista Sovietikal

1

LD : /décident / de promouvoir / la coopération, / dans un esprit/

$$
2 \quad 3 \quad 4 \quad 5
$$

LA : /dia tapa-kevitra / hanentana / ny fiaraha-miasa/ araka ny toe-tsainal

LD : /de compréhension mutuelle/ afin de contribuer/ au développement/

LA : /samy ifankahazoana / mba handraisan'anjara / amin'ny fampandrosoanal

LD : /économique / et technique / des deux pays / en vue de réaliser/

$9010 \quad 11$ a 12

LA : Iny toe-karena / sy ny fahaiza-manao zavatra / ao amin'ny firenena roa tontal Mba hanatontosoanal

LD : /(cette) coopération.../ les deux Gouvernements/

$13 \quad 14$ LA : /izany fiaraha-miasa (izany)... / dia samy hifanampy/

LD : /se prêteront assistance,/ notamment / par la voie de.../

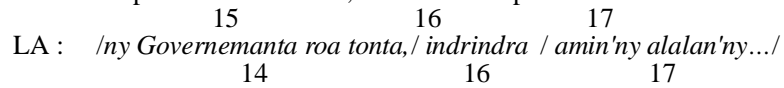


Litt. : «Le Gouvernement de la République Malgache et le Gouvernement de l'URSS décident de former [sens moderne] la coopération, selon l'état d'esprit où ils s'entendront mutuellement ensemble, afin de prendre part au développement de l'économie [état de la richesse] et du savoir-faire dans les deux nations. En vue d'accomplir cette coopération, les deux gouvernements s'entraideront ensemble, notamment par la voie de ...» (Accord de Coopération Économique et Technique entre le Gouvernement de la République Malgache et le Gouvernement de l'URSS, J.O.R.D.M. du 01/11/75).

14. E. Coseriu (1967) : Structures lexicales et enseignement du vocabulaire. Les théories linguistiques et leurs applications, Didier, p. 347.

15. Texte original français : «les deux gouvernements se prêteront assistance, notamment par la voie [...] de l'octroi de crédits destinés au financement de programmes de recherche et de développement : travaux de prospection».

16. Litt. «comme le peuple venant de la nation qui a eu le plus d'avantages».

17. Litt. «comme ce qu'obtiennent le peuple et les citoyens venant de la nation qui a eu le plus d'avantages».

18. Litt. «ce qui est permis à la nation préférée».

19. Il y est stipulé, en effet, que «les terres malgaches ne peuvent être vendues ni hypothéquées aux étrangers ni à qui que ce soit, excepté entre les Malgaches, et celui qui les vendrait ou les hypothéquerait serait condamné aux fers à perpétuité». 